\title{
Gerontoneurologie und Gerontopsychiatrie im Fokus
}

\author{
Liebe Kolleginnen, liebe Kollegen,
}

Wie wir alle wissen, steuert die Alterspyramide in Deutschland in die falsche Richtung, d.h. wir haben es mit einer zunehmend alten Gesellschaft zu tun. Dies bedeutet andererseits aber auch, dass wir - Neurologen und Psychiater - in der Bedeutung der Krankenversorgung einen ständig zunehmenden Einfluss und einen hohen Stellenwert einnehmen.

Es kann davon ausgegangen werden, dass in den nächsten Dekaden etwa 50\% aller stationär zu betreuenden Patienten aus dem Gebiet der neuropsychiatrischen Erkrankungen kommen werden (Quelle: Max-Planck-Institut für Demoskopie, Rostock). Aus diesem Grunde ist es auch wichtig, dass eine konsequente Fortbildung auf dem Gebiet der Gerontoneurologie und der Gerontopsychiatrie erfolgt.

Wir wissen alle, dass die großen Krankheitsbilder wie Demenz, Depressionen, Schlaganfälle, Morbus Parkinson zu den wichtigsten Krankheitsbildern unserer Fächer gehören. Somit ist es sehr erfreulich, dass wir die Möglichkeit eingeräumt bekamen, in der psychoneuro zu diesen Krankheitsbildern ein Schwerpunktheft verfassen zu dürfen. Wir beschäftigen uns dabei mit den Schlaganfällen, der Epilepsie im Alter, den Gangstörungen, neu- ropsychologischen Untersuchungsverfahren sowie der Alzheimer-Demenz.

Wichtig ist es mir, darauf hinzuweisen, dass neben Gerontoneurologie und Gerontopsychiatrie nicht lediglich die „normale“ Verordnung von Medikamenten beim alten Menschen beinhaltet, sondern dass hier erhebliche Interaktionen mit Medikamenten ausgelöst werden können, die bei Patienten jüngeren Alters so nicht gesehen werden. Es ist somit stets zu beachten, ob ein älterer Patient eine gestörte Leber- oder Nierenfunktion aufweist; Blutdruck und die kardiale Situation müssen mit ins Kalkül gezogen werden und viele psychotrope Medikamente können zu schwerstwiegenden Nebenwirkungen wie Delir oder Halluzinationen führen.

Es ist wichtig, dass der Facharzt für Neurologie und Psychiatrie einen besonderen Zugang zu diesen Situationen beim alten Menschen gewinnt. Dementsprechend haben wir in unseren Artikeln gerade auf diese Sichtweise besonderen Wert gelegt. Ich freue mich, dass sich so namhafte Autoren der Herausforderung gestellt haben, in übersichtlicher, kurzer und einprägsamer Weise diese wichtigen Themen zu bearbeiten. Ich bin optimistisch, dass unsere Anstrengungen bei den Lesern auf positive Resonanz treffen werden.

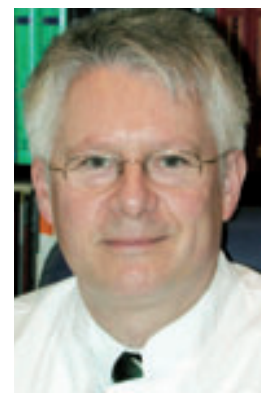

Prof. Heinz Reichmann, Dresden 